\title{
Nausea and vomiting in gastroparesis: similarities and differences in idiopathic and diabetic gastroparesis
}

\author{
H. P. PARKMAN, ${ }^{\star}$ E. K. HALlinAN, $\dagger$ W. L. HASLER, $\uparrow$ G. FARRUGIA, $§$ K. L. KOCH, $\uparrow$ J. CALLES, $\uparrow$ W. J. SNAPE, ${ }^{\star}$

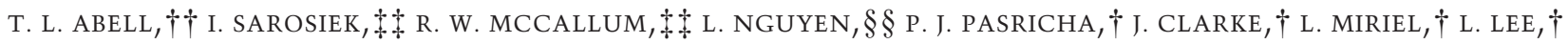 \\ J. TONASCIA, $\uparrow \&$ F. HAMILTON $\mid \boldsymbol{\uparrow}$, THE NIDDK GASTROPARESIS CLINICAL RESEARCH CONSORTIUM (GPCRC) \\ ^Temple University, Philadelphia, PA, USA \\ $\dagger$ Johns Hopkins University, Baltimore, MD, USA \\ †niversity of Michigan, Ann Arbor, MI, USA \\ §Mayo Clinic, Rochester, MN, USA \\ qWake Forest University, Winston-Salem, NC, USA \\ ${ }^{\star \star}$ California Pacific Medical Center, San Francisco, CA, USA \\ $\dagger \dagger$ University of Louisville, Louisville, KY, USA \\ †Texas Tech University, El Paso, TX, USA \\ $\S \S$ Stanford University, Palo Alto, CA, USA \\ qqNational Institute of Diabetes and Digestive and Kidney Diseases, Bethesda, MD, USA
}

\section{Key Points}

- Nausea and vomiting are classic symptoms in patients with gastroparesis. Most studies combine nausea and vomiting into one symptom complex; there may be different characteristics relating to nausea as compared vomiting. There may be different characteristics of these symptoms in diabetic compared to idiopathic gastroparesis.

- Nausea is present in essentially all patients with gastroparesis irrespective of cause. Nausea is associated with decreased quality of life in patients with gastroparesis. Vomiting was more prevalent, more severe, and occurred more often in diabetic compared to idiopathic gastroparesis.

- The characteristics of vomiting differ in idiopathic vs diabetic gastroparesis.

\begin{abstract}
Background Nausea and vomiting are classic symptoms of gastroparesis. It remains unclear if characteristics of nausea and vomiting are similar in different etiologies of gastroparesis. The aims of this article were as follows: to describe characteristics of nausea and vomiting in patients with gastroparesis and to determine if there are differences in nausea and vomiting in diabetic (DG) and idiopathic gastroparesis
\end{abstract}

Address for Correspondence

Henry P. Parkman, MD, Gastroenterology Section, Temple University School of Medicine, Parkinson Pavilion, 8th floor, 3401 North Broad Street, Philadelphia, PA 19140, USA

Tel: +1 215707 7579; fax: +1 215707 2684;

e-mail: henry.parkman@temple.edu

Received: 24 March 2016

Accepted for publication: 30 May 2016
(IG). Methods Gastroparetic patients enrolling in the NIDDK Gastroparesis Registry underwent assessment with history and questionnaires assessing symptoms, quality of life, and a questionnaire characterizing nausea and vomiting. Key Results Of 159 gastroparesis patients (107 IG, 52 DG), 96\% experienced nausea, whereas $65 \%$ experienced vomiting. Nausea was predominant symptom in $28 \%$ and vomiting was predominant in 4\%. Nausea was severe or very severe in 41\%. PAGI-SYM nausea/vomiting subscore was greater with increased vomiting severity, but not nausea severity in DG than IG. Nausea was related to meals in $71 \%$; lasting most of the day in $41 \%$. Increasing nausea severity was related to decreased quality of life. Nausea often preceded vomiting in $82 \%$ of patients and vomiting often relieved nausea in 30\%. Vomiting was more common in DG (81\%) compared 
to IG $(57 \% ; p=0.004)$. Diabetic patients more often had vomiting in the morning before eating, during the night, and when not eating. Conclusions \& Inferences Nausea is present in essentially all patients with gastroparesis irrespective of cause and associated with decreased quality of life. In contrast, vomiting was more prevalent, more severe, and occurred more often in DG than IG. Thus, characteristics of vomiting differ in $I G$ vs $D G$.

Keywords diabetic gastroparesis, gastric emptying, gastroparesis, Nausea, vomiting.

\section{INTRODUCTION}

Nausea and vomiting are classic symptoms in patients with gastroparesis. ${ }^{1}$ While most patients experience some degree of nausea, only some gastroparesis patients have vomiting with some studies suggest vomiting is seen in less than $50 \%$ of patients with gastroparesis. ${ }^{2}$ Studies have suggested that nausea and vomiting symptoms correlate with worse quality of life in gastroparesis patients. ${ }^{3,4}$ Despite the importance of nausea and vomiting in gastroparesis, the characteristics of these symptoms have not been well described.

Nausea and vomiting may have different manifestations in different etiologies of gastroparesis. Some studies have suggested that nausea and vomiting are more severe in diabetic gastroparesis (DG) than idiopathic gastroparesis (IG). ${ }^{3-5}$ Most studies combine nausea and vomiting into one symptom complex; there may be different characteristics relating to nausea as compared vomiting. There may be different characteristics of these symptoms in diabetic compared to IG. The potential differential perception of nausea in diabetic vs IG might be due to different pathophysiological mechanisms and the effects of diabetes on neuronal function. This has important treatment implications for nausea and vomiting in patients with diabetic and IG.

The aims of this study were to describe characteristics of nausea as compared to vomiting in patients with gastroparesis and to determine if there are differences between two etiologies of gastroparesis DG and IG. We also aimed to better understand the relationship between nausea and vomiting in gastroparesis, determine if nausea and vomiting impact on the impaired quality of life in gastroparesis, and investigate the relationship of nausea and vomiting with gastric emptying.

\section{METHODS}

\section{Overview}

The NIDDK Gastroparesis Clinical Research Consortium is a cooperative network of eight academic motility centers and one Data Coordinating Center (DCC). ${ }^{5,6}$ The Gastroparesis Registry 2 (ClinicalTrials.gov Identifier: NCT01696747) was implemented as an observational study of patients with gastroparesis enrolled prospectively at eight centers. This study uses data from the second gastroparesis registry, which was designed, in part, to enhance the understanding of symptoms and physiologic dysfunction in patients with gastroparesis. There was a special emphasis to look at the symptoms of nausea and vomiting through a Nausea and Vomiting Questionnaire which was designed to assess the clinical characteristics of both nausea and vomiting.

\section{Study patients}

Gastroparetic patients were enrolled at eight centers into the NIH Gastroparesis Registry from September 2012 to August 2015. Enrolled patients met specific entry criteria being 18 years or older with symptoms of at least 12 weeks duration, delayed gastric emptying scintigraphy (GES) within 6 months of enrollment, and no structural abnormality as seen by upper endoscopy within 1 year of enrollment.

This report focuses on patients with either idiopathic or DG. The diabetic patients could have either type 1 diabetes mellitus (T1DM) or type 2 diabetes mellitus (T2DM) as defined by the physician and/or patient. The diagnosis of patients with the idiopathic etiology was based on no previous gastric surgery, no diabetes history (before or after the onset of gastroparesis at enrollment), a normal hemoglobin $\mathrm{Al}_{\mathrm{C}}$, and no other known etiologies.

All studies were approved by the Institutional Review Board at each Clinical Center and at the DCC.

\section{Study protocol}

During face-to-face interviews with each subject, the study physicians or coordinators at each Clinical Center completed case report forms including data relating to gastroparesis disease onset, symptoms, disease profile, associated medical conditions, including diabetes, and medication and supplemental therapies. The study physicians performed a comprehensive physical examination. Laboratory measures were obtained, including hemoglobin $\mathrm{A} 1_{\mathrm{C}}$ values, antinuclear antibody (ANA), and erythrocyte sedimentation rate (ESR).

The clinical severity of gastroparesis was graded on a scale originally reported in the American Neurogastroenterology and Motility Society (ANMS) review on treatment of gastroparesis. ${ }^{7}$ The severity was graded as grade 1: mild gastroparesis (symptoms relatively easily controlled and able to maintain weight and nutrition on a regular diet); grade 2: compensated gastroparesis (moderate symptoms with only partial control with use of daily medications, able to maintain nutrition with dietary adjustments); grade 3: gastroparesis with gastric failure (refractory symptoms that are not controlled as shown by the patient having ER visits, frequent doctor visits or hospitalizations and/or inability to maintain nutrition via an oral route). 
Each patient filled out the 20 item Patient Assessment of Upper Gastrointestinal Symptoms (PAGI-SYM) questionnaire which assesses symptoms of gastroparesis, dyspepsia, and gastroesophageal reflux disease ${ }^{8}$; it includes the nine symptoms of the Gastroparesis Cardinal Symptom Index (GCSI) which asks about nausea, retching, vomiting, stomach fullness, inability to finish a meal, excessive fullness, loss of appetite, bloating, and abdominal distension. ${ }^{9}$ The GCSI equals the mean of the nausea/ vomiting subscore, postprandial fullness/early satiety subscore, and bloating subscore where: Nausea/vomiting subscore $=$ mean of the scores for nausea, retching, and vomiting; Postprandial fullness/early satiety sub-score $=$ mean of the scores for stomach fullness, inability to finish meal, excessive fullness, and loss of appetite; and Bloating subscore = mean of the scores for bloating and large stomach. The PAGI-SYM also inquires about symptoms of gastroesophageal reflux including daytime heartburn, heartburn lying down, daytime chest discomfort, nighttime chest discomfort, daytime reflux, nighttime reflux, and bitter taste. In the PAGI-SYM, patients are asked to assess the severity of their symptoms during the previous 2 weeks using a $0-5$ scale where no symptoms $=0$, very mild $=1$, mild $=2$, moderate $=3$, severe $=4$, and very severe $=5$.

Disease-specific quality of life was assessed by the Patient Assessment of Upper Gastrointestinal Disorders-Quality of Life (PAGI-QOL) survey, which scores 30 factors from 0 (none of the time) to 5 (all of the time). ${ }^{10}$ Patients were asked how often gastrointestinal problems they may be experiencing have affected different aspects of their quality of life and well-being in the past 2 weeks. Overall PAGI-QOL scores were calculated by taking means of all subscores after reversing item scores; thus a mean PAGI-QOL score of 0 represents poor quality of life, whereas 5 reflects the best life quality.

The Medical Outcomes Study 36-Item Short-Form Health Survey version 2 (SF-36v2) was additionally used to assess the patients' views of overall physical and mental health in the past 4 weeks (standard recall form). The eight subscales were standardized to the 1998 U.S. general population with a mean $( \pm$ SD $)$ of $50 \pm 10$. Physical and mental health summary measures were computed. A higher score reflects higher quality of life. ${ }^{11}$

A Nausea and Vomiting Questionnaire was designed to assess the clinical characteristics of both nausea and vomiting. Part of this questionnaire is a modification of the Nausea Profile characterizing nausea in three dimensions: somatic distress, GI distress, and emotional distress. ${ }^{12}$ This questionnaire had previously been modified for capturing nausea related to gastroparesis. ${ }^{3}$

\section{Gastric emptying scintigraphy}

Gastric emptying scintigraphy was performed using a low-fat, egg white meal with imaging at $0,1,2,4 \mathrm{~h}$ after meal ingestion, as described by a published multicenter protocol ${ }^{13}$ and endorsed by the Society of Nuclear Medicine and ANMS. ${ }^{14}$ This protocol ensures standardized information about gastric emptying across sites. In addition, liquid gastric emptying in the presence of solids was assessed using Indium-111. ${ }^{15}$

Patients were instructed to stop medications that could affect gastrointestinal motility for the $72 \mathrm{~h}$ prior to the study and to come to the Nuclear Medicine Section in the morning after fasting overnight with nothing to eat after midnight, that is, an $8 \mathrm{~h}$ fast. Gastric emptying scintigraphy was performed using a standard low-fat, Eggbeaters ${ }^{\circledR}$ meal to measure solid emptying. ${ }^{13,14}$ The meal consisted of the equivalent of two large eggs radiolabeled with Tc-99m sulfur colloid served with two pieces of white bread and jelly. In addition, patients were given $120 \mathrm{~mL}$ water radiolabeled with In-111 diethylene triamine pentacetic acid for the measurement of liquid gastric emptying. Following ingestion of the meal, imaging was performed at $0,1,2,3$ and $4 \mathrm{~h}$ with the patient upright for measuring gastric emptying of Tclabeled solids and 111-In-labeled liquids. In between imaging, patients generally sat in the nuclear medicine waiting area.

Gastric emptying was analyzed as the percent of radioactivity retained in the stomach over time using the geometric center of the decay-corrected anterior and posterior counts for each time point. Gastric retention of Tc- $99 \mathrm{~m}>60 \%$ at $2 \mathrm{~h}$ and/or $>10 \%$ at $4 \mathrm{~h}$ was considered evidence of delayed gastric emptying of solids. Delayed gastric emptying was graded according to the gastric retention at $4 \mathrm{~h}$ : mild $(\leq 20 \%$ gastric retention at $4 \mathrm{~h})$, moderate $(>20-35 \%)$, and severe $(>35 \%) .{ }^{14,16}$ Delayed gastric emptying of liquids in the presence of solids is greater than $50 \%$ retention of In-111 at $1 \mathrm{~h}$ emptying. ${ }^{15}$

\section{Statistical methods}

Descriptive statistics (means, standard deviations, frequencies, and percentages) were used to compare subgroups of gastroparesis patients. Enrollment characteristics such as demographics, medical history, gastroparesis history, symptom severity, and quality of life were compared by etiology (idiopathic compared to diabetic). $P$-values were determined from Fisher's exact tests for categorical variables and $t$-tests for continuous variables. Enrollment characteristics were also compared by the subgroups of nausea severity score on the PAGI-SYM instrument (none/very $\mathrm{mild} / \mathrm{mild}$, moderate, and severe/very severe) and the subgroup of vomiting severity score on the PAGI-SYM instrument (none, very mild/mild/moderate, and severe/very severe). $P$-values were determined from a Cochran-Armitage test for trend in nausea or vomiting subgroups for binary variables, a Mantel-Haenszel chisquared test for trend in nausea or vomiting subgroups for categorical variables, and a non-parametric Cuzick test for trend in nausea or vomiting subgroups for continuous variables. ${ }^{17}$ Multiple logistic models were selected based on Akaike Information criteria using forward selection of all possible models derived from a candidate set of 16 enrollment variables (see Table 1). ${ }^{18,19}$ The resulting model for severe nausea included etiology, age, solid gastric emptying percent at $4 \mathrm{~h}$, PAGI-SYM satiety/fullness subscore, SF-36 mental score, SF-36 physical score, and PAGIQOL score. The resulting model for severe vomiting included etiology, age, race, PAGI-QOL score, $\mathrm{HbAlc} \%$, and the following PAGI-SYM measures: satiety/fullness subscore, bloating subscore, and GERD subscore. All p-values are two-sided; values $<0.05$ were considered statistically significant. Analyses were performed using methods described in SAS version 9.3 (SAS Institute) or Stata version 13.1 (StataCorp). ${ }^{20}$

\section{RESULTS}

\section{Patient characteristics}

One hundred and fifty-nine patients with gastroparesis were evaluated: 107 patients with IG and 52 patients with DG ( 35 with T1DM, 17 with T2DM). Average age was $44.7 \pm 13.3$ years. Females comprised the majority of patients $(84.9 \%)$. Table 1 contains other demographic information. The majority of patients had compensated (grade 2) gastroparesis (66.0\%) with moderate severity of symptoms of gastroparesis (GCSI 
Table 1 Characteristics of patients with idiopathic or diabetic gastroparesis

\begin{tabular}{|c|c|c|c|c|}
\hline \multirow[b]{2}{*}{ Characteristic } & \multicolumn{2}{|l|}{ Etiology } & \multirow[b]{2}{*}{ Total $(n=159)$} & \multirow[b]{2}{*}{$p$-value ${ }^{\dagger}$} \\
\hline & Idiopathic $(n=107)$ & Diabetic $(n=52)$ & & \\
\hline \multicolumn{5}{|l|}{ Demographics } \\
\hline Gender: females & $97(90.7 \%)$ & $38(73.1 \%)$ & $135(84.9 \%)$ & 0.008 \\
\hline Age (years) & $43.6 \pm 14.0$ & $46.9 \pm 11.8$ & $44.7 \pm 13.3$ & 0.14 \\
\hline Hispanic & $9(8.4 \%)$ & $13(25.0 \%)$ & $22(13.8 \%)$ & 0.007 \\
\hline Race: white & $98(91.6 \%)$ & $42(80.8 \%)$ & $140(88.1 \%)$ & 0.07 \\
\hline \multicolumn{5}{|l|}{ Gastroparesis history } \\
\hline Duration of symptoms (years) & $6.0 \pm 6.5$ & $8.1 \pm 7.8$ & $6.7 \pm 7.0$ & 0.08 \\
\hline \multicolumn{5}{|l|}{ Onset of gastroparesis symptoms } \\
\hline Acute & $46(43.0 \%)$ & $20(38.5 \%)$ & $66(41.5 \%)$ & \multirow[t]{2}{*}{0.50} \\
\hline Insidious or gradual & $59(55.1 \%)$ & $32(61.5 \%)$ & $91(57.2 \%)$ & \\
\hline \multicolumn{5}{|l|}{ Predominant symptom prompting gastroparesis evaluation } \\
\hline Nausea & $37(34.6 \%)$ & $11(21.2 \%)$ & $48(30.2 \%)$ & \multirow[t]{4}{*}{0.26} \\
\hline Vomiting & $15(14.0 \%)$ & $8(15.4 \%)$ & $23(14.5 \%)$ & \\
\hline Abdominal pain & $24(22.4 \%)$ & $11(21.2 \%)$ & $35(22.0 \%)$ & \\
\hline Other & $31(29.0 \%)$ & $22(42.3 \%)$ & $53(33.3 \%)$ & \\
\hline \multicolumn{5}{|l|}{ Nature of gastroparesis symptoms } \\
\hline Chronic, but stable & $20(18.9 \%)$ & $6(11.5 \%)$ & $26(16.5 \%)$ & \multirow[t]{6}{*}{0.70} \\
\hline Chronic, but progressive worsening & $22(20.8 \%)$ & $11(21.2 \%)$ & $33(20.9 \%)$ & \\
\hline Chronic, but some improvement & $10(9.4 \%)$ & $8(15.4 \%)$ & $18(11.4 \%)$ & \\
\hline Chronic with periodic exacerbations & $37(34.9 \%)$ & $17(32.3 \%)$ & $54(34.2 \%)$ & \\
\hline Cyclic pattern & $16(15.1 \%)$ & $9(17.3 \%)$ & $25(15.8 \%)$ & \\
\hline Asymptomatic & $1(0.9 \%)$ & $1(1.9 \%)$ & $2(1.3 \%)$ & \\
\hline \multicolumn{5}{|l|}{ Gastroparesis severity } \\
\hline Mild (grade 1) & $20(18.7 \%)$ & $12(23.1 \%)$ & $32(20.1 \%)$ & \multirow[t]{3}{*}{0.47} \\
\hline Compensated (grade 2) & $74(69.2 \%)$ & $31(59.6 \%)$ & $105(66.0 \%)$ & \\
\hline Gastric failure (grade 3) & $13(12.2 \%)$ & $9(17.3 \%)$ & $22(13.8 \%)$ & \\
\hline \multicolumn{5}{|l|}{ Weight history } \\
\hline BMI $\left(\mathrm{kg} / \mathrm{m}^{2}\right)$ & $26.5 \pm 8.2$ & $29.3 \pm 6.7$ & $27.4 \pm 7.8$ & 0.03 \\
\hline Medical history & & & & \\
\hline Diabetes & & & & \\
\hline Type 1 & & $35(67.3 \%)$ & & \\
\hline Type 2 & & $17(32.7 \%)$ & & \\
\hline Hospitalization for gastroparesis in the past year & $22(20.6 \%)$ & $22(42.3 \%)$ & $44(27.7 \%)$ & 0.004 \\
\hline Number of hospitalizations for gastroparesis in the past year & $2.4 \pm 1.7$ & $7.0 \pm 11.0$ & $4.7 \pm 8.2$ & 0.06 \\
\hline Use of $\mathrm{G}$ tube & $3(2.8 \%)$ & $0(0.0 \%)$ & $3(1.9 \%)$ & 0.55 \\
\hline Use of J tube & $2(1.9 \%)$ & $1(1.9 \%)$ & $3(1.9 \%)$ & 1.00 \\
\hline Presence of central line & $0(0.0 \%)$ & $4(7.7 \%)$ & $4(2.7 \%)$ & 0.01 \\
\hline Presence of gastric stimulator & $5(4.7 \%)$ & $10(19.2 \%)$ & $15(9.4 \%)$ & 0.007 \\
\hline Use of prokinetics & $34(31.8 \%)$ & $22(42.3 \%)$ & $56(35.2 \%)$ & 0.19 \\
\hline Use of Botox (ever) & $28(26.2 \%)$ & $19(36.5 \%)$ & $47(29.6 \%)$ & 0.20 \\
\hline Use of antinausea medications & $87(81.3 \%)$ & $42(80.8 \%)$ & $129(81.1 \%)$ & 1.00 \\
\hline Use of narcotics & $87(35.5 \%)$ & $20(38.5 \%)$ & $58(36.5 \%)$ & 0.72 \\
\hline Use of alternative medications & $53(49.5 \%)$ & $12(23.1 \%)$ & $65(40.9 \%)$ & 0.002 \\
\hline PAGI-SYM symptom severity $(0-5)^{*}$ & & & & \\
\hline Nausea score & $2.9 \pm 1.6$ & $3.0 \pm 1.6$ & $2.9 \pm 1.6$ & 0.64 \\
\hline Vomiting score & $0.9 \pm 1.4$ & $1.9 \pm 1.8$ & $1.2 \pm 1.6$ & 0.0001 \\
\hline Retching score & $1.1 \pm 1.5$ & $1.8 \pm 1.7$ & $1.4 \pm 1.6$ & 0.01 \\
\hline Nausea subscore & $1.6 \pm 1.2$ & $2.3 \pm 1.5$ & $1.8 \pm 1.4$ & 0.006 \\
\hline Satiety subscore & $3.3 \pm 1.2$ & $3.3 \pm 1.3$ & $3.3 \pm 1.2$ & 0.88 \\
\hline Bloating subsore & $3.1 \pm 1.6$ & $3.0 \pm 1.7$ & $3.0 \pm 1.6$ & 0.88 \\
\hline Cardinal symptom index (GCSI) & $2.7 \pm 1.1$ & $2.9 \pm 1.1$ & $2.7 \pm 1.1$ & 0.27 \\
\hline Upper abdominal pain subscore & $2.8 \pm 1.6$ & $2.8 \pm 1.5$ & $2.8 \pm 1.5$ & 0.93 \\
\hline GERD subscore & $1.8 \pm 1.3$ & $1.8 \pm 1.5$ & $1.8 \pm 1.4$ & 0.89 \\
\hline Predominant symptom of PAGI-SYM & & & & \\
\hline Nausea & $31(29.0 \%)$ & $13(25.0 \%)$ & $44(27.7 \%)$ & 0.15 \\
\hline Vomiting & $5(4.7 \%)$ & $2(3.9 \%)$ & $7(4.4 \%)$ & \\
\hline Upper abdominal pain or discomfort & $18(16.8 \%)$ & $3(5.8 \%)$ & $21(13.2 \%)$ & \\
\hline Other & $53(49.5 \%)$ & $34(65.4 \%)$ & $87(54.7 \%)$ & \\
\hline PAGI-QOL $(0-5)^{\S}$ & & & & \\
\hline Activity subscore & $2.6 \pm 1.2$ & $2.9 \pm 1.2$ & $2.7 \pm 1.2$ & 0.29 \\
\hline Clothing subscore & $2.9 \pm 1.7$ & $3.0 \pm 1.9$ & $2.9 \pm 1.7$ & 0.58 \\
\hline
\end{tabular}


Table 1 (continued)

\begin{tabular}{|c|c|c|c|c|}
\hline \multirow[b]{2}{*}{ Characteristic } & \multicolumn{2}{|l|}{ Etiology } & \multirow[b]{2}{*}{ Total $(n=159)$} & \multirow[b]{2}{*}{$p$-value } \\
\hline & Idiopathic $(n=107)$ & Diabetic $(n=52)$ & & \\
\hline Diet subscore & $1.6 \pm 1.3$ & $2.2 \pm 1.4$ & $1.8 \pm 1.3$ & 0.006 \\
\hline Relationship subscore & $3.4 \pm 1.2$ & $3.3 \pm 1.5$ & $3.3 \pm 1.3$ & 0.56 \\
\hline Psychology subscore & $3.3 \pm 1.3$ & $3.1 \pm 1.5$ & $3.2 \pm 1.4$ & 0.39 \\
\hline Total PAGI-QOL & $2.8 \pm 1.0$ & $2.9 \pm 1.3$ & $2.8 \pm 1.1$ & 0.47 \\
\hline \multicolumn{5}{|l|}{ SF-36v2 Health Survey (past 4 weeks) ${ }^{\pi}$} \\
\hline Physical health summary measure & $33.7 \pm 9.7$ & $33.8 \pm 11.4$ & $33.7 \pm 10.2$ & 0.94 \\
\hline Mental health summary measure & $43.0 \pm 13.5$ & $41.1 \pm 13.8$ & $42.4 \pm 13.6$ & 0.41 \\
\hline \multicolumn{5}{|l|}{ Solid gastric scintigraphy } \\
\hline Percent retention at $1 \mathrm{~h}$ & $79.3 \pm 13.8$ & $80.8 \pm 12.6$ & $79.8 \pm 13.3$ & 0.51 \\
\hline Percent retention at $2 \mathrm{~h}$ & $63.0 \pm 16.3$ & $64.7 \pm 19.0$ & $63.6 \pm 17.2$ & 0.58 \\
\hline Percent retention at $4 \mathrm{~h}$ & $26.5 \pm 16.5$ & $37.1 \pm 22.3$ & $30.0 \pm 19.1$ & 0.0009 \\
\hline \multicolumn{5}{|l|}{ Liquid gastric scintigraphy } \\
\hline Percent retention at $30 \mathrm{~min}$ & $63.1 \pm 17.3$ & $68.6 \pm 17.4$ & $65.0 \pm 17.4$ & 0.19 \\
\hline Percent retention at $1 \mathrm{~h}$ & $49.1 \pm 16.6$ & $50.8 \pm 19.7$ & $49.7 \pm 17.6$ & 0.66 \\
\hline
\end{tabular}

Nausea/vomiting severity is a subscale from the Patient Assessment of Upper Gastrointestinal Disorders Symptom Severity Index (PAGI-SYM). It is the average of the nausea, retching, and vomiting severity scores. ${ }^{\top}$ The significance of difference in categorical variables between groups was tested with a chi-squared test or Fisher's exact test. Continuous variables were analyzed with a $t$-test. All $p$-values are two-sided. ${ }^{*}$ Subscales derived from the Patient Assessment of Upper Gastrointestinal Disorders Symptom Severity Index (PAGI-SYM). A higher score reflects a greater severity. ${ }^{\S}$ Subscales derived from the Patient Assessment of Upper Gastrointestinal Disorders-Quality of Life (PAGI-QOL). Scales have been recoded so that a higher score reflects a higher QOL. "Scores on the Medical Outcomes Study 36-Item Short-Form Health Survey V2 (SF-36v2) standard recall were normalized to the 1998 U.S. general population with a mean $( \pm S D)$ of $50 \pm 10$. A higher score reflects higher QOL or better health outcome. Data are means \pm SD or number (percents).

score of $2.7 \pm 1.1)$. However, $13.8 \%$ of patients were graded as having gastric failure with $27.7 \%$ of these patients having been hospitalized within last year. Symptoms prompting evaluation for gastroparesis included nausea $(30.2 \%)$, vomiting $(14.5 \%)$, and abdominal pain $(22.0 \%)$. At the time of enrollment in the registry, the predominant symptoms were nausea in $27.7 \%$ of patients, upper abdominal pain in $13.2 \%$ and vomiting in $4.4 \%$ of patients. Antinausea medications were being used by $81.1 \%$ of the patients, prokinetics agent use in $35.2 \%$, and narcotic analgesics by $36.5 \%$. Other treatments included gastric electric stimulator in $9.4 \%$ of patients, use of $\mathrm{G}$ tube in $1.9 \%$, use of $\mathrm{J}$ tube in $1.9 \%$, and presence of a central line in $2.7 \%$. Overall the gastric emptying was moderately delayed with $30.0 \%$ retention at $4 \mathrm{~h}$, being more delayed in DG $(37.1 \%$ retention) than IG $(26.5 \%$ retention; $p=0.0009$ ). For the diabetic patients, the average HgbAlc was $8.3 \pm 2.0 \%$ with $53.9 \%$ of the diabetic patients having HgbAlc $\geq 8.0 \%$. There was a decreased quality of life in the patients with gastroparesis most prominently with the SF-36 physical score being 33.7 compared to normal of 50 .

\section{Nausea/Vomiting severity using PAGI-SYM}

Table 1 compares the PAGI-SYM symptom severity between diabetic and idiopathic patients. The nausea/ vomiting subscore of the PAGI-SYM (average of nausea, retching, and vomiting severity) was greater in diabetic $(2.3 \pm 1.5 ; p=0.006)$ than idiopathics $(1.6 \pm 1.2)$ with increased vomiting severity in diabetic $(1.9 \pm 1.8 ; p=0.0001)$ than idiopathic $(0.9 \pm 1.4)$ and increased retching severity in diabetics $(1.8 \pm 1.7)$ than idiopathic $(1.1 \pm 1.5 ; p=0.01)$. Nausea severity was not different between IG and DG $3.0 \pm 1.6$ for diabetic vs $2.9 \pm 1.6$ for idiopathic $(p=0.64)$.

Table 2 shows characteristics of patients with gastroparesis according to their nausea severity as assessed using the PAGI-SYM. Nausea severity was severe or very severe in 65 of $159(41 \%)$ patients (42 of 107 [40\%] IG and 23 of 52 [45\%] IG; $p=0.77)$. The severity of retching and vomiting increased as nausea severity increased. The severity of other symptoms of gastroparesis also tracked with the severity of nausea: satiety/fullness subscore $(p<0.0001)$, bloating subscore $(p=0.002)$, upper abdominal pain subscore $(p<0.0001)$, and GERD subscore $(p=0.03)$.

Increasing nausea was related to decreased quality of life by PAGI-QOL $(p=0.005)$, especially in the activity subscore $(p<0.001)$, diet subscore $(p=0.005)$, and relationship subscore $(p=0.01)$. Increasing nausea was associated with decreased quality of life using the SF-36: SF-36 physical $(p=0.01)$ and mental $(p=0.03)$ measures.

There was a trend for increasing antiemetic use $(p=0.04)$ and narcotic use $(p=0.06)$ with increasing nausea severity. In the diabetic patients, there were 
Table 2 Characteristics of patients with idiopathic or diabetic gastroparesis by nausea severity

\begin{tabular}{|c|c|c|c|c|c|}
\hline \multirow[b]{2}{*}{ Characteristic } & \multicolumn{3}{|c|}{ Nausea severity on PAGI-SYM* ${ }^{\star}$} & \multirow[b]{2}{*}{$\begin{array}{l}\text { Total } \\
(n=159)\end{array}$} & \multirow[b]{2}{*}{$p$-value ${ }^{\dagger}$} \\
\hline & $\begin{array}{l}\text { None/very } \\
\text { mild/mild } \\
(n=51)\end{array}$ & $\begin{array}{l}\text { Moderate } \\
(n=43)\end{array}$ & $\begin{array}{l}\text { Severe/very } \\
\text { severe } \\
(n=65)\end{array}$ & & \\
\hline \multicolumn{6}{|l|}{ Etiology } \\
\hline Idiopathic & $34(66.7 \%)$ & $31(72.1 \%)$ & $42(64.6 \%)$ & $107(67.3 \%)$ & \multirow[t]{2}{*}{0.77} \\
\hline Diabetic & $17(33.3 \%)$ & $12(27.9 \%)$ & $23(35.4 \%)$ & $52(32.7 \%)$ & \\
\hline \multicolumn{6}{|l|}{ Demographics } \\
\hline Gender: females & $45(88.3 \%)$ & $35(81.4 \%)$ & $55(84.6 \%)$ & $135(84.9 \%)$ & 0.62 \\
\hline Age (years) & $48.3 \pm 13.8$ & $43.0 \pm 13.4$ & $41.0 \pm 12.1$ & $44.7 \pm 13.3$ & 0.001 \\
\hline Hispanic & $11(21.6 \%)$ & $3(7.0 \%)$ & $8(12.3 \%)$ & $22(13.8 \%)$ & 0.18 \\
\hline Race: white & $49(96.1 \%)$ & $38(88.4 \%)$ & $53(81.5 \%)$ & $140(88.1 \%)$ & 0.02 \\
\hline \multicolumn{6}{|l|}{ Gastroparesis history } \\
\hline \multicolumn{6}{|l|}{ Nature of gastroparesis symptoms } \\
\hline Chronic, but stable & $10(19.6 \%)$ & $7(16.3 \%)$ & $9(14.1 \%)$ & $26(16.5 \%)$ & \multirow[t]{6}{*}{0.95} \\
\hline Chronic, but progressive worsening & $6(11.8 \%)$ & $11(25.6 \%)$ & $16(25.0 \%)$ & $33(20.9 \%)$ & \\
\hline Chronic, but some improvement & $11(21.6 \%)$ & $4(9.3 \%)$ & $3(4.7 \%)$ & $18(11.4 \%)$ & \\
\hline Chronic with periodic exacerbations & $15(29.4 \%)$ & $13(30.2 \%)$ & $26(40.6 \%)$ & $54(34.2 \%)$ & \\
\hline Cyclic pattern & $7(13.7 \%)$ & $8(18.6 \%)$ & $10(15.6 \%)$ & $25(15.8 \%)$ & \\
\hline Asymptomatic & $2(3.9 \%)$ & $0(0.0 \%)$ & $0(0.0 \%)$ & $2(1.3 \%)$ & \\
\hline \multicolumn{6}{|l|}{ Gastroparesis severity } \\
\hline Mild (grade 1) & $22(43.1 \%)$ & $3(7.0 \%)$ & $7(10.8 \%)$ & $32(20.1 \%)$ & \multirow{3}{*}{$<0.0001$} \\
\hline Compensated (grade 2) & $27(52.9 \%)$ & $34(79.1 \%)$ & $44(67.7 \%)$ & $105(66.0 \%)$ & \\
\hline Gastric failure (grade 3) & $2(3.9 \%)$ & $6(14.0 \%)$ & $14(21.5 \%)$ & $22(13.8 \%)$ & \\
\hline \multicolumn{6}{|l|}{ Medical history } \\
\hline BMI $\left(\mathrm{kg} / \mathrm{m}^{2}\right)$ & $27.8 \pm 8.8$ & $28.3 \pm 6.4$ & $26.5 \pm 7.9$ & $27.4 \pm 7.8$ & 0.30 \\
\hline Use of prokinetics & $14(27.5 \%)$ & $17(39.5 \%)$ & $25(38.5 \%)$ & $56(35.2 \%)$ & 0.24 \\
\hline Use of antiemetics & $37(72.6 \%)$ & $35(81.4 \%)$ & $57(87.7 \%)$ & $129(81.1 \%)$ & 0.04 \\
\hline Use of narcotics & $15(29.4 \%)$ & $13(30.2 \%)$ & $30(46.2 \%)$ & $58(36.5 \%)$ & 0.06 \\
\hline \multicolumn{6}{|l|}{ Laboratory results } \\
\hline HbAlc, if diabetic (\%) & $8.3 \pm 2.0$ & $8.2 \pm 2.4$ & $8.3 \pm 1.8$ & $8.3 \pm 2.0$ & 0.91 \\
\hline $\mathrm{HbAlc} \geq 8.0 \%$, if diabetic & $11(64.7 \%)$ & $5(41.7 \%)$ & $12(52.2 \%)$ & $28(53.9 \%)$ & 0.48 \\
\hline ANA negative, if idiopathic & $26(76.5 \%)$ & $29(93.6 \%)$ & $36(85.7 \%)$ & $91(85.1 \%)$ & 0.30 \\
\hline ESR, if idiopathic & $15.6 \pm 13.0$ & $15.3 \pm 10.3$ & $12.8 \pm 12.6$ & $14.4 \pm 12.0$ & 0.28 \\
\hline ESR elevated $>30 \mathrm{~mm}$, if idiopathic & $6(17.7 \%)$ & $4(12.9 \%)$ & $2(5.0 \%)$ & $12(11.4 \%)$ & 0.09 \\
\hline \multicolumn{6}{|l|}{ PAGI-SYM symptom severity $(0-5)^{*}$} \\
\hline Nausea score & $0.9 \pm 0.9$ & $3.0 \pm 0.0$ & $4.4 \pm 0.5$ & $2.9 \pm 1.6$ & $<0.001$ \\
\hline Vomiting score & $0.4 \pm 0.9$ & $1.2 \pm 1.3$ & $1.9 \pm 1.9$ & $1.2 \pm 1.6$ & $<0.001$ \\
\hline Retching score & $0.4 \pm 0.8$ & $1.3 \pm 1.2$ & $2.1 \pm 1.8$ & $1.4 \pm 1.6$ & $<0.001$ \\
\hline Nausea/vomiting subscore & $0.5 \pm 0.7$ & $1.8 \pm 0.7$ & $2.8 \pm 1.2$ & $1.8 \pm 1.4$ & $<0.001$ \\
\hline Satiety/fullness subscore & $2.5 \pm 1.3$ & $3.3 \pm 0.9$ & $3.9 \pm 1.0$ & $3.3 \pm 1.2$ & $<0.001$ \\
\hline Bloating subscore & $2.4 \pm 1.8$ & $3.2 \pm 1.4$ & $3.4 \pm 1.5$ & $3.0 \pm 1.6$ & 0.002 \\
\hline Cardinal symptom index (GCSI) & $1.8 \pm 1.0$ & $2.8 \pm 0.7$ & $3.4 \pm 0.8$ & $2.7 \pm 1.1$ & $<0.001$ \\
\hline Upper abdominal pain subscore & $2.0 \pm 1.7$ & $2.7 \pm 1.2$ & $3.4 \pm 1.4$ & $2.8 \pm 1.5$ & $<0.001$ \\
\hline GERD subscore & $1.4 \pm 1.4$ & $2.1 \pm 1.3$ & $2.0 \pm 1.4$ & $1.8 \pm 1.4$ & 0.03 \\
\hline \multicolumn{6}{|c|}{ Predominant symptom from the PAGI-SYM } \\
\hline Nausea & $2(3.9 \%)$ & $12(27.9 \%)$ & $30(46.2 \%)$ & $44(27.7 \%)$ & 0.08 \\
\hline Vomiting & $1(2.0 \%)$ & $1(2.3 \%)$ & $5(7.7 \%)$ & $7(4.4 \%)$ & \\
\hline Upper abdominal pain or discomfort & $6(11.8 \%)$ & $4(9.3 \%)$ & $11(16.9 \%)$ & $21(13.2 \%)$ & \\
\hline Other & $42(82.4 \%)$ & $26(60.5 \%)$ & $19(29.2 \%)$ & $87(54.7 \%)$ & \\
\hline PAGI-QOL $(0-5)^{\S}$ & & & & & \\
\hline Activity subscore & $3.2 \pm 1.1$ & $2.6 \pm 1.1$ & $2.3 \pm 1.2$ & $2.7 \pm 1.2$ & $<0.001$ \\
\hline Clothing subscore & $3.2 \pm 1.8$ & $2.8 \pm 1.5$ & $2.8 \pm 1.9$ & $2.9 \pm 1.7$ & 0.27 \\
\hline Diet subscore & $2.3 \pm 1.5$ & $1.7 \pm 1.2$ & $1.5 \pm 1.2$ & $1.8 \pm 1.3$ & 0.005 \\
\hline Relationship subscore & $3.7 \pm 1.3$ & $3.2 \pm 1.3$ & $3.1 \pm 1.3$ & $3.3 \pm 1.3$ & 0.01 \\
\hline Psychology subscore & $3.4 \pm 1.3$ & $3.3 \pm 1.2$ & $2.9 \pm 1.5$ & $3.2 \pm 1.4$ & 0.09 \\
\hline Total PAGI-QOL & $3.2 \pm 1.1$ & $2.7 \pm 1.0$ & $2.5 \pm 1.1$ & $2.8 \pm 1.1$ & 0.005 \\
\hline SF-36v2 Health Survey (past 4 weeks) & & & & & \\
\hline Physical health summary measure & $36.3 \pm 10.9$ & $34.4 \pm 9.8$ & $31.3 \pm 9.5$ & $33.7 \pm 10.2$ & 0.01 \\
\hline Mental health summary measure & $44.7 \pm 13.5$ & $44.1 \pm 12.9$ & $39.4 \pm 13.7$ & $42.4 \pm 13.6$ & 0.03 \\
\hline Solid gastric scintigraphy & & & & & \\
\hline Percent retention at $2 \mathrm{~h}$ & $63.6 \pm 17.2$ & $59.7 \pm 15.6$ & $66.2 \pm 18.0$ & $63.6 \pm 17.2$ & 0.27 \\
\hline Percent retention at $4 \mathrm{~h}$ & $29.5 \pm 19.8$ & $23.5 \pm 14.2$ & $34.6 \pm 20.4$ & $30.0 \pm 19.2$ & 0.09 \\
\hline
\end{tabular}


Table 2 (continued)

\begin{tabular}{|c|c|c|c|c|c|}
\hline \multirow[b]{2}{*}{ Characteristic } & \multicolumn{3}{|c|}{ Nausea severity on PAGI-SYM* } & \multirow[b]{2}{*}{$\begin{array}{l}\text { Total } \\
|n=159|\end{array}$} & \multirow[b]{2}{*}{$p$-value } \\
\hline & $\begin{array}{l}\text { None/very } \\
\text { mild/mild } \\
(n=51)\end{array}$ & $\begin{array}{l}\text { Moderate } \\
(n=43)\end{array}$ & $\begin{array}{l}\text { Severe/very } \\
\text { severe } \\
(n=65)\end{array}$ & & \\
\hline Percent retention at $4 \mathrm{~h}$, diabetic patients only & $37.1 \pm 26.7$ & $32.7 \pm 19.3$ & $39.5 \pm 20.7$ & $37.1 \pm 22.3$ & 0.33 \\
\hline Percent retention at $4 \mathrm{~h}$, idiopathic patients only & $25.7 \pm 14.4$ & $20.0 \pm 9.9$ & $31.9 \pm 20.0$ & $26.5 \pm 16.5$ & 0.17 \\
\hline \multicolumn{6}{|l|}{ Liquid gastric scintigraphy } \\
\hline Percent retention at $30 \mathrm{~min}$ & $64.1 \pm 16.9$ & $67.3 \pm 14.8$ & $63.8 \pm 20.0$ & $65.0 \pm 17.4$ & 0.83 \\
\hline Percent retention at $1 \mathrm{~h}$ & $49.2 \pm 19.0$ & $52.2 \pm 13.6$ & $48.1 \pm 19.4$ & $49.7 \pm 17.6$ & 0.36 \\
\hline
\end{tabular}

${ }^{\star}$ Nausea severity is a score from the Patient Assessment of Upper Gastrointestinal Disorders Symptom Severity Index (PAGI-SYM). ${ }^{\dagger}$ The significance of difference in binary variables between groups was tested with a Cochran-Armitage trend test, the significance of difference in categorical variables between groups was tested with a Mantel-Haenszel chi-squared test, and the significance of difference in continuous variables between groups was tested with a non-parametric Cuzick test for trend. All p-values are two-sided. ${ }^{\ddagger}$ Subscales derived from the Patient Assessment of Upper Gastrointestinal Disorders Symptom Severity Index (PAGI-SYM). A higher score reflects a greater severity. ${ }^{\S}$ Subscales derived from the Patient Assessment of Upper Gastrointestinal Disorders-Quality of Life (PAGI-QOL). Scales have been recoded so that a higher score reflects a higher QOL. "Scores on the Medical Outcomes Study 36-Item Short-Form Health Survey V2 (SF-36v2) standard recall were normalized to the 1998 U.S. general population with a mean $( \pm \mathrm{SD})$ of $50 \pm 10$. A higher score reflects higher QOL or better health outcome. Data are means $\pm \mathrm{SD}$ or number (percents).

similar HgbAlc values across the different severities of nausea.

Severe or very severe nausea patients had increased gastric retention at $4 \mathrm{~h}$ on the GES test $(34.6 \%$ retention for severe/very severe compared to $23.5 \%$ for moderate, and $29.5 \%$ none $/$ mild; $p=0.09 \mid$. Severity of nausea was not related to retention of liquids $(p=0.36)$.

Table 3 shows characteristics of patients according to their vomiting severity. Vomiting was present at the time of enrollment in 75 of 159 patients $(48 \%)$, being present more often in DG $(65 \%)$ than in IG $(38 \%$; $p=0.002)$. Percentage wise, more patients with DG (11 of 52 or $21 \%$ ) had severe/very severe vomiting compared to IG (12 of 107 or $11 \%$; $p=0.15)$. As expected, increasing retching and nausea severity were seen with increasing vomiting severity. Increasing vomiting severity tracked with other symptoms of gastroparesis; satiety subscore $(p<0.001)$, bloating subscore $(p=0.002)$, upper abdominal pain subscore $(p<0.001)$, and GERD subscore $(p=0.03)$. Increasing vomiting severity was associated with worsening quality of life on the PAGI-QOL ( $p=0.005)$, especially activity $(p<0.001)$, relationship $(p=0.01)$ subscores. Increasing vomiting was associated with decreased SF36 physical component $(p=0.01)$ and mental component $(p=0.03)$. In diabetic patients, HgbAlc tended to be higher in those with more severe vomiting $(9.0 \pm 1.9 \%)$, but the trend was not significant $(p=0.81)$. Use of prokinetic agents, antiemetic agents, and narcotic analgesics increased with increasing vomiting severity. Retention at $4 \mathrm{~h}$ on GES differed, but not statistically significantly, in the vomiting severity subgroups $(p=0.09)$ : with $39.8 \%$ retention in those with severe/very severe vomiting, compared to $26.6 \%$ retention for those with mild/moderate vomiting, and $29.4 \%$ retention for those with no vomiting.

We further looked at the relationship of gastroparetic symptoms with delayed gastric emptying (Table S1). Gastric retention at $4 \mathrm{~h}$ was greater in diabetic than IG. More patients with DG had severe gastric retention than IG. Stomach fullness and postprandial fullness, but not nausea and vomiting, were significantly increased with increasing gastric retention at $4 \mathrm{~h}$ using symptoms captured at enrollment. We also collected symptoms at time of the gastric emptying test. As with surveys obtained on enrollment, symptom severities measured at the time of the gastric emptying test showed no significant relation of nausea or vomiting to gastric retention rates. Increasing stomach fullness was associated with increasing gastric retention. Use of antiemetics, but not prokinetic or narcotic analgesics, was associated with more severe retention during gastric emptying testing.

The gastroparesis patients were also compared according to the 2 and $4 \mathrm{~h}$ gastric emptying data by dividing the patients in three groups: (i) Delayed at $2 \mathrm{~h}$, normal at $4 \mathrm{~h}$; (ii) Delayed at $2 \mathrm{~h}$, delayed at $4 \mathrm{~h}$; and (iii) Normal at $2 \mathrm{~h}$, delayed at $4 \mathrm{~h}$. The severity of nausea, retching, early satiety, and upper abdominal pain were similar among these groups.

Most patients had nausea. There were 24 patients scoring no nausea on the PAGI-SYM, 84 patients with no vomiting on the PAGI-SYM, and 23 patients with no nausea or vomiting. Using the nausea and vomiting form, there were six with no nausea, 56 with no vomiting, and six with no nausea or vomiting. The patients with no nausea or vomiting on the PAGI-SYM 
Table 3 Characteristics of patients with idiopathic or diabetic gastroparesis by vomiting severity

\begin{tabular}{|c|c|c|c|c|c|}
\hline \multirow[b]{2}{*}{ Characteristic } & \multicolumn{3}{|c|}{ Vomiting severity ${ }^{\star}$} & \multirow[b]{2}{*}{$\begin{array}{l}\text { Total } \\
(n=159)\end{array}$} & \multirow[b]{2}{*}{$p$-value ${ }^{\dagger}$} \\
\hline & $\begin{array}{l}\text { None } \\
(n=84)\end{array}$ & $\begin{array}{l}\text { Very } \\
\text { mild/mild/ } \\
\text { moderate } \\
(n=52)\end{array}$ & $\begin{array}{l}\text { Severe/very } \\
\text { severe } \\
(n=23)\end{array}$ & & \\
\hline \multicolumn{6}{|l|}{ Etiology } \\
\hline Idiopathic & $66(78.6 \%)$ & $29(55.8 \%)$ & $12(52.2 \%)$ & $107(67.3 \%)$ & \multirow[t]{2}{*}{0.003} \\
\hline Diabetic & $18(21.4 \%)$ & $23(44.2 \%)$ & $11(47.8 \%)$ & $52(32.7 \%)$ & \\
\hline \multicolumn{6}{|l|}{ Demographics } \\
\hline Gender: females & $75(89.3 \%)$ & $41(78.9 \%)$ & $19(82.6 \%)$ & $135(84.9 \%)$ & 0.20 \\
\hline Age (years) & $45.4 \pm 14.1$ & $45.2 \pm 12.4$ & $41.0 \pm 12.5$ & $44.7 \pm 13.3$ & 0.001 \\
\hline Hispanic & $11(13.1 \%)$ & $7(13.5 \%)$ & $4(17.4 \%)$ & $22(13.8 \%)$ & 0.65 \\
\hline Race: white & $77(91.7 \%)$ & $47(90.4 \%)$ & $16(69.6 \%)$ & $140(88.1 \%)$ & 0.01 \\
\hline \multicolumn{6}{|l|}{ Medical history } \\
\hline \multicolumn{6}{|l|}{ Nature of gastroparesis symptoms } \\
\hline Chronic, but stable & $19(22.6 \%)$ & $6(11.8 \%)$ & $1(4.4 \%)$ & $26(16.5 \%)$ & \multirow[t]{6}{*}{0.17} \\
\hline Chronic, but progressive worsening & $14(16.7 \%)$ & $13(25.5 \%)$ & $6(26.1 \%)$ & $33(20.9 \%)$ & \\
\hline Chronic, but some improvement & $8(9.5 \%)$ & $9(17.7 \%)$ & $1(4.4 \%)$ & $18(11.4 \%)$ & \\
\hline Chronic with periodic exacerbations & $31(36.9 \%)$ & $14(27.5 \%)$ & $9(39.1 \%)$ & $54(34.2 \%)$ & \\
\hline Cyclic pattern & $10(11.9 \%)$ & $9(17.7 \%)$ & $6(26.1 \%)$ & $25(15.8 \%)$ & \\
\hline Asymptomatic & $2(2.4 \%)$ & $0(0.0 \%)$ & $0(0.0 \%)$ & $2(1.3 \%)$ & \\
\hline \multicolumn{6}{|l|}{ Gastroparesis severity } \\
\hline Mild (grade 1) & $25(29.8 \%)$ & $6(11.5 \%)$ & $1(4.4 \%)$ & $32(20.1 \%)$ & \multirow[t]{3}{*}{$<0.0001$} \\
\hline Compensated (grade 2) & $54(64.3 \%)$ & $37(71.2 \%)$ & $14(60.9 \%)$ & $105(66.1 \%)$ & \\
\hline Gastric failure (grade 3) & $5(6.0 \%)$ & $9(17.3 \%)$ & $8(34.8 \%)$ & $22(13.8 \%)$ & \\
\hline \multicolumn{6}{|l|}{ Medical history } \\
\hline BMI $\left(\mathrm{kg} / \mathrm{m}^{2}\right)$ & $27.7 \pm 8.6$ & $28.1 \pm 6.9$ & $24.8 \pm 6.3$ & $27.4 \pm 7.8$ & 0.30 \\
\hline Use of prokinetics & $21(25.0 \%)$ & $21(40.4 \%)$ & $14(60.9 \%)$ & $56(35.2 \%)$ & 0.0009 \\
\hline Use of antiemetics & $63(75.0 \%)$ & $45(86.5 \%)$ & $21(91.3 \%)$ & $129(81.1 \%)$ & 0.04 \\
\hline Use of narcotics & $21(25.0 \%)$ & $23(44.2 \%)$ & $14(60.9 \%)$ & $58(36.5 \%)$ & 0.0005 \\
\hline \multicolumn{6}{|l|}{ Laboratory results } \\
\hline HbAlc, if diabetic $(\%)$ & $8.5 \pm 1.7$ & $7.8 \pm 2.1$ & $9.0 \pm 1.9$ & $8.3 \pm 2.0$ & 0.81 \\
\hline $\mathrm{HbAlc} \geq 8.0 \%$, if diabetic & $12(66.7 \%)$ & $9(39.1 \%)$ & $7(63.6 \%)$ & $28(53.9 \%)$ & 0.64 \\
\hline ANA negative, if idiopathic & $54(81.8 \%)$ & $25(86.2 \%)$ & $12(100.0 \%)$ & $91(85.1 \%)$ & 0.12 \\
\hline ESR, if idiopathic & $15.6 \pm 12.9$ & $12.0 \pm 10.0$ & $13.7 \pm 11.6$ & $14.4 \pm 12.0$ & 0.39 \\
\hline ESR elevated $>30 \mathrm{~mm}$, if idiopathic & $9(13.9 \%)$ & $2(6.9 \%)$ & $1(9.1 \%)$ & $12(11.4 \%)$ & 0.41 \\
\hline \multicolumn{6}{|l|}{ PAGI-SYM symptom severity $(0-5)^{\star}$} \\
\hline Nausea score & $2.3 \pm 1.7$ & $3.3 \pm 1.2$ & $4.4 \pm 0.9$ & $2.9 \pm 1.6$ & $<0.001$ \\
\hline Vomiting score & $0.0 \pm 0.0$ & $1.8 \pm 0.8$ & $4.4 \pm 0.5$ & $1.2 \pm 1.6$ & $<0.001$ \\
\hline Retching score & $0.4 \pm 0.9$ & $2.1 \pm 1.4$ & $3.3 \pm 1.4$ & $1.4 \pm 1.6$ & $<0.001$ \\
\hline Nausea/vomiting subscore & $0.9 \pm 0.7$ & $2.4 \pm 0.9$ & $4.0 \pm 0.6$ & $1.8 \pm 1.4$ & $<0.001$ \\
\hline Satiety/fullness subscore & $3.0 \pm 1.3$ & $3.5 \pm 0.9$ & $3.9 \pm 1.2$ & $3.3 \pm 1.2$ & $<0.001$ \\
\hline Bloating subsore & $2.8 \pm 1.6$ & $3.3 \pm 1.5$ & $3.4 \pm 1.7$ & $3.0 \pm 1.6$ & 0.002 \\
\hline Cardinal symptom index (GCSI) & $2.2 \pm 1.0$ & $3.1 \pm 0.8$ & $3.8 \pm 0.9$ & $2.7 \pm 1.1$ & $<0.001$ \\
\hline Upper abdominal pain subscore & $2.4 \pm 1.6$ & $3.2 \pm 1.3$ & $3.2 \pm 1.5$ & $2.8 \pm 1.5$ & $<0.001$ \\
\hline GERD subscore & $1.5 \pm 1.3$ & $2.0 \pm 1.4$ & $2.6 \pm 1.5$ & $1.8 \pm 1.4$ & 0.03 \\
\hline \multicolumn{6}{|l|}{ Predominant symptom from the PAGI-SYM } \\
\hline Nausea & $20(23.8 \%)$ & $15(28.9 \%)$ & $9(39.1 \%)$ & $44(27.7 \%)$ & 0.13 \\
\hline Vomiting & $0(0.0 \%)$ & $0(0.0 \%)$ & $7(30.4 \%)$ & $7(4.4 \%)$ & \\
\hline Upper abdominal pain or discomfort & $12(14.3 \%)$ & $8(15.4 \%)$ & $1(4.4 \%)$ & $21(13.2 \%)$ & \\
\hline Other & $52(61.9 \%)$ & $29(55.8 \%)$ & $6(26.1 \%)$ & $87(54.7 \%)$ & \\
\hline PAGI-QOL $(0-5)^{\S}$ & & & & & \\
\hline Activity subscore & $2.9 \pm 1.1$ & $2.6 \pm 1.2$ & $2.1 \pm 1.1$ & $2.7 \pm 1.2$ & $<0.001$ \\
\hline Clothing subscore & $3.1 \pm 1.7$ & $2.8 \pm 1.7$ & $2.6 \pm 2.1$ & $2.9 \pm 1.7$ & 0.27 \\
\hline Diet subscore & $1.9 \pm 1.4$ & $1.8 \pm 1.3$ & $1.3 \pm 1.1$ & $1.8 \pm 1.3$ & 0.005 \\
\hline Relationship subscore & $3.6 \pm 1.2$ & $3.1 \pm 1.5$ & $2.9 \pm 1.4$ & $3.3 \pm 1.3$ & 0.01 \\
\hline Psychology subscore & $3.4 \pm 1.2$ & $3.1 \pm 1.5$ & $2.7 \pm 1.5$ & $3.2 \pm 1.4$ & 0.09 \\
\hline Total PAGI-QOL & $3.0 \pm 1.0$ & $2.7 \pm 1.1$ & $2.3 \pm 1.1$ & $2.8 \pm 1.1$ & 0.005 \\
\hline SF-36v2 Health Survey (past 4 weeks) & & & & & \\
\hline Physical health summary measure & $35.5 \pm 9.6$ & $32.0 \pm 11.1$ & $31.1 \pm 9.8$ & $33.7 \pm 10.2$ & 0.01 \\
\hline Mental health summary measure & $42.7 \pm 13.3$ & $42.8 \pm 14.5$ & $40.2 \pm 12.5$ & $42.4 \pm 13.6$ & 0.03 \\
\hline Solid gastric scintigraphy & & & & & \\
\hline Percent retention at $1 \mathrm{~h}$ & $79.9 \pm 14.4$ & $80.6 \pm 10.6$ & $77.8 \pm 15.1$ & $79.8 \pm 13.3$ & 0.69 \\
\hline
\end{tabular}


Table 3 (continued)

\begin{tabular}{|c|c|c|c|c|c|}
\hline \multirow[b]{2}{*}{ Characteristic } & \multicolumn{3}{|c|}{ Vomiting severity* } & \multirow[b]{2}{*}{$\begin{array}{l}\text { Total } \\
(n=159)\end{array}$} & \multirow[b]{2}{*}{$p$-value } \\
\hline & $\begin{array}{l}\text { None } \\
(n=84)\end{array}$ & $\begin{array}{l}\text { Very } \\
\text { mild/mild/ } \\
\text { moderate } \\
(n=52)\end{array}$ & $\begin{array}{l}\text { Severe/very } \\
\text { severe } \\
(n=23)\end{array}$ & & \\
\hline Percent retention at $2 \mathrm{~h}$ & $64.8 \pm 16.9$ & $61.2 \pm 16.5$ & $64.7 \pm 20.1$ & $63.6 \pm 17.2$ & 0.27 \\
\hline Percent retention at $4 \mathrm{~h}$ & $29.4 \pm 19.4$ & $26.6 \pm 14.7$ & $39.8 \pm 24.2$ & $30.0 \pm 19.1$ & 0.09 \\
\hline Percent retention at $4 \mathrm{~h}$, diabetic patients only & $40.0 \pm 28.9$ & $33.5 \pm 16.7$ & $39.9 \pm 21.1$ & $37.1 \pm 22.3$ & 0.78 \\
\hline Percent retention at $4 \mathrm{~h}$, idiopathic patients only & $26.5 \pm 14.9$ & $21.0 \pm 10.3$ & $39.7 \pm 27.6$ & $26.5 \pm 16.5$ & 0.65 \\
\hline \multicolumn{6}{|l|}{ Liquid gastric scintigraphy } \\
\hline Percent retention at $30 \mathrm{~min}$ & $64.1 \pm 16.7$ & $67.3 \pm 13.6$ & $62.8 \pm 24.7$ & $65.0 \pm 17.4$ & 0.83 \\
\hline Percent retention at $1 \mathrm{~h}$ & $48.2 \pm 17.3$ & $50.7 \pm 13.4$ & $51.5 \pm 25.7$ & $49.7 \pm 17.6$ & 0.36 \\
\hline
\end{tabular}

${ }^{\star}$ Vomiting severity is a score from the Patient Assessment of Upper Gastrointestinal Disorders Symptom Severity Index (PAGI-SYM). ${ }^{\dagger}$ The significance of difference in binary variables between groups was tested with a Cochran-Armitage trend test, the significance of difference in categorical variables between groups was tested with a Mantel-Haenszel chi-squared test, and the significance of difference in continuous variables

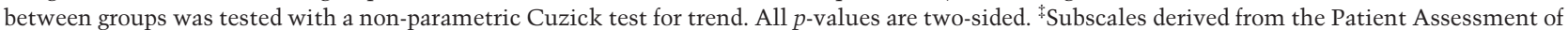
Upper Gastrointestinal Disorders Symptom Severity Index (PAGI-SYM). A higher score reflects a greater severity. ${ }^{8}$ Subscales derived from the Patient Assessment of Upper Gastrointestinal Disorders-Quality of Life (PAGI-QOL). Scales have been recoded so that a higher score reflects a higher QOL. "Scores on the Medical Outcomes Study 36-Item Short-Form Health Survey V2 (SF-36v2) standard recall were normalized to the 1998 U.S. general population with a mean $( \pm \mathrm{SD})$ of $50 \pm 10$. A higher score reflects higher QOL or better health outcome. Data are means $\pm \mathrm{SD}$ or number (percents).

\begin{tabular}{|c|c|c|c|c|c|c|}
\hline \multirow{2}{*}{$\begin{array}{l}\text { Enrollment } \\
\text { characteristic }\end{array}$} & \multicolumn{3}{|c|}{ Unadjusted analyses } & \multicolumn{3}{|c|}{ Adjusted analyses } \\
\hline & OR & $\mathrm{CI}$ & $p^{\dagger}$ & OR & CI & $p^{*}$ \\
\hline Etiology (diabetic vs idiopathic) & 1.23 & $(0.63,2.40)$ & 0.55 & 1.12 & $(0.48,2.61)$ & 0.80 \\
\hline Age, years & 0.96 & $(0.94,0.99)$ & 0.004 & 0.96 & $(0.93,0.99)$ & 0.005 \\
\hline $\begin{array}{l}\text { Solid gastric emptying } \\
\text { scintigraphy, percent retention at } 4 \mathrm{~h}\end{array}$ & 1.02 & $(1.00,1.04)$ & 0.01 & 1.02 & $(0.99,1.04)$ & 0.14 \\
\hline PAGI-SYM, satiety/fullness subscore & 2.58 & $(1.76,3.77)$ & $<0.001$ & 2.83 & $(1.80,4.44)$ & $<0.001$ \\
\hline SF-36, mental score & 0.97 & $(0.95,1.00)$ & 0.02 & 0.94 & $(0.90,0.98)$ & 0.006 \\
\hline SF-36, physical score & 0.96 & $(0.93,0.99)$ & 0.01 & 0.95 & $(0.90,0.99)$ & 0.02 \\
\hline PAGI-QOL score & 0.70 & $(0.52,0.94)$ & 0.02 & 1.75 & $(0.95,3.20)$ & 0.07 \\
\hline
\end{tabular}

Table 4 Logistic regression analyses of enrollment characteristics as predictors of severe nausea ${ }^{\star}$ in idiopathic and diabetic gastroparetics $(n=159)$

*Severe nausea defined as 'severe' or 'very severe' nausea score on the PAGI-SYM instrument. 'Unadjusted odds ratios, 95\% confidence limits, $p$-values determined from logistic regression models of severe nausea on each predictor. ${ }^{*}$ Adjusted odds ratios, $95 \%$ confidence limits, $p$ values were determined from a multiple logistic regression analyses of severe nausea using all baseline predictors indicated. This model was determined from Akaike Information criteria (AIC) with forward selection using a candidate set of baseline variables: gender, age at enrollment, etiology, race, SF-36 physical score, SF-36 mental score, PAGI-QOL total score, solid GES $2 \mathrm{~h}$ retention percent, solid GES $4 \mathrm{~h}$ retention percent, HbAlc, ESR, gastroparesis severity, and the following PAGI-SYM items: satiety subscore, bloating subscore, upper abdominal pain subscore, and GERD subscore. Etiology was forced into the model.

had a higher BMI than patients with nausea and/or vomiting $(32 \pm 11$ vs $27 \pm 7 ; p=0.004)$. There were also significantly less other gastroparesis symptoms on the PAGI-SYM including satiety subscore $(2.3 \pm 1.4 \mathrm{vs}$ $3.5 \pm 1.1 ; \quad p<0.0001)$ and upper abdominal pain $(1.8 \pm 1.8$ vs $3.0 \pm 1.4 ; p=0.001)$. There were similar percentages of diabetic/idiopathic patients and similar percent retention at 2 and $4 \mathrm{~h}$ between these two groups.

Logistic regression analysis was used to look at independent predictors of nausea and vomiting severity (Tables 4 and 5). Severe/very severe nausea according to the PAGI-SYM was associated with younger age, increased satiety subscore, decreased mental SF-36 score, and decreased SF-36 physical score (Table 4). Severe vomiting was associated with non-white race, increased satiety subscore, decreased bloating subscore, and increased GERD subscore (Table 5).

\section{Characteristics of nausea/vomiting}

The characteristics of nausea and vomiting are shown Table S2. Overall 153 of 159 patients (96.2\%) experienced nausea as a symptom $197.2 \%$ of idiopathics and $94.2 \%$ of diabetic patients). The nausea was lasting most of the day $(41.2 \%)$ or at least several hours of the 
Table 5 Logistic regression analyses of enrollment characteristics as predictors of severe vomiting ${ }^{\star}$ in idiopathic and diabetic gastroparetics $(n=159)$

\begin{tabular}{|c|c|c|c|c|c|c|}
\hline \multirow[b]{2}{*}{ Enrollment characteristic } & \multicolumn{3}{|c|}{ Unadjusted analyses } & \multicolumn{3}{|c|}{ Adjusted analyses } \\
\hline & OR & CI & $p^{\dagger}$ & OR & CI & $p^{\ddagger}$ \\
\hline Etiology (diabetic vs idiopathic) & 2.12 & $(0.87,5.21)$ & 0.10 & 1.02 & $(0.19,5.59)$ & 0.98 \\
\hline Age, years & 0.98 & $(0.94,1.01)$ & 0.16 & 0.97 & $(0.93,1.01)$ & 0.16 \\
\hline Race (white vs non-white) & 0.22 & $(0.08,0.64)$ & 0.006 & 0.20 & $(0.05,0.80)$ & 0.02 \\
\hline PAGI-SYM, satiety subscore & 1.95 & $(1.18,3.21)$ & 0.009 & 2.17 & $(1.19,3.95)$ & 0.01 \\
\hline PAGI-SYM, bloating subscore & 1.19 & $(0.89,1.60)$ & 0.24 & 0.62 & $(0.41,0.95)$ & 0.03 \\
\hline PAGI-SYM, GERD subscore & 1.60 & $(1.15,2.22)$ & 0.005 & 1.77 & $(1.16,2.72)$ & 0.009 \\
\hline PAGI-QOL score & 0.63 & $(0.42,0.95)$ & 0.03 & 0.71 & $(0.42,1.20)$ & 0.20 \\
\hline HbAlc, \% & 1.28 & $(1.04,1.59)$ & 0.02 & 1.29 & $(0.85,1.95)$ & 0.24 \\
\hline
\end{tabular}

*Severe vomiting defined as 'severe' or 'very severe' vomiting score on the PAGI-SYM instrument. "Unadjusted odds ratios, 95\% confidence limits, $p$ values determined from logistic regression models of severe vomiting on each predictor. ${ }^{\star}$ Adjusted odds ratios, $95 \%$ confidence limits, $p$-values were determined from a multiple logistic regression analyses of severe nausea using all baseline predictors indicated. This model was determined from Akaike Information criteria (AIC) with forward selection using a candidate set of baseline variables: gender, age at enrollment, etiology, race, SF-36 physical score, SF-36 mental score, PAGI-QOL total score, solid GES $2 \mathrm{~h}$ retention percent, solid GES $4 \mathrm{~h}$ retention percent, HbAlc, ESR, gastroparesis severity, and the following PAGI-SYM items: satiety subscore, bloating subscore, upper abdominal pain subscore, and GERD subscore. Etiology was forced into the model.

day $(27.5 \%)$; whereas in $31.4 \%$ of the patients, the nausea lasted for about an hour or less. Nausea was related to meals in $71.2 \%$, but felt by patients to be unrelated to eating in $28.8 \%$. Nausea was worse in the morning before eating in $27.5 \%$ of patients and worse in the evening in $26.1 \%$ of patients. Other factors that were related to increasing nausea included high fat meals $(44.4 \%$ of patients), dairy (32.0\% of patients), being hungry $(26.3 \%)$, riding in a car $(25.7 \%)$. There were no significant differences in these characteristics of nausea between patients with DG and IG. Nausea increasing during or after meals tended to be more frequently reported by IG $(52.4 \%)$ compared to DG $(32.7 \% ; p=0.06)$.

The nausea profile was compared between patients with idiopathic and DG. The total nausea profile was not significantly different between idiopathic and diabetic patients (46.4 vs 46.5 ; $p=0.99$ ); with similar values for the somatic, GI distress, and emotional distress subscales.

The characteristics of vomiting are also shown in Table S2. Overall $64.8 \%$ of patients experienced vomiting as a symptom, being experienced more in diabetic $(80.8 \%)$ compared to idiopathic patients $157.0 \%$ of idiopathic patients; $p=0.004)$. Vomiting lasted for several minutes in $51.0 \%$ of patients, about $30 \mathrm{~min}$ to several hours in $32.4 \%$, and most of the day in $16.7 \%$ of patients; tending to be more prolonged in diabetic than idiopathic patients $(p=0.11)$. Vomiting often was related to eating $(72.8 \%$ of patients), being unrelated to eating in $27.2 \%$. The vomitus was described as partial digested food in $45.4 \%$ or undigested food in $34.0 \%$. Vomiting occurred in the morning before eating more often in diabetic $(69.0 \%)$ than idiopathic patients $(44.3 \% ; p=0.04)$. The vomiting could wake patients up at night in $55.4 \%$ of patients, being more prevalent in DG than IG $(p=0.02)$. Nausea often preceded vomiting in $81.6 \%$ of patients; whereas vomiting often relieved nausea in $30.1 \%$. Vomiting could occur even if no food or drink was take in $35.0 \%$ of patients, being more common in diabetic $(45.2 \%)$, then idiopathic patients $(27.9 \%$; $p=0.008)$.

\section{DISCUSSION}

This study has carefully detailed the characteristics of both nausea and vomiting in patients with gastroparesis; two important, and often considered classical symptoms of gastroparesis. This study finds that nausea is present in nearly all $(96 \%)$ patients with gastroparesis. Nausea was the predominant symptom in $28 \%$ of the patients, the most common of the single individual symptoms. Nausea was present for many hours in the majority of patients. The characteristics of nausea (severity, timing) were similar in diabetic and idiopathic patients. Vomiting was present in approximately half the patients but was considered the predominant symptoms in only a small percentage $(4 \%)$ of the patients. In contrast to nausea, vomiting was more prevalent and severe in diabetic than in IG.

This study documents the decreased quality of life in patients with gastroparesis. The SF-36 physical score was 33.7 compared to normal of 50 . There was less effect on the mental quality of life with SF-36 of 42.4. Increasing nausea and vomiting were both related to decreased quality of life using the disease-specific instrument PAGI-QOL. Using logistic regression analysis, nausea severity, but not vomiting severity, was independently associated with the SF-36 QOL scores. Thus, gastroparesis has an increased clinical burden as demonstrated objectively by decreased quality of life, 
and nausea severity is associated with this decreased quality of life. Other smaller studies have shown that nausea and vomiting symptoms are associated with impaired quality of life, ${ }^{3,4}$ but this study importantly separates the characteristics of nausea and vomiting.

Nausea was present in nearly all patients with gastroparesis, irrespective of the etiology. Nausea was generally present for many hours in the majority of patients. The characteristics of nausea (severity, timing) were similar in diabetic and idiopathic patients. In contrast, vomiting was less prevalent, being present in roughly half of patients with gastroparesis, with significant differences in the characteristics of vomiting among the diabetic and idiopathic patients. Vomiting was more common and more severe in patients with diabetic than IG. Interestingly, diabetic patients more often had vomiting occurring in the morning before eating, during the night, and could occur even if the patient did not eat. Clinically, many patients state they do not want to vomit and limit their intake and change diet so that do not have vomiting. On the other hand, some patients find that vomiting helps to relieve the nausea. Our prior study also suggested that nausea and vomiting were more severe in diabetic than IG. ${ }^{21}$ This study expands this by showing it is the vomiting characteristics that appear to be different between diabetic and IG with the nausea being somewhat similar between the two. The vomiting data was assessed by PAGI-SYM and our nausea and vomiting questionnaire. In the PAGISYM, vomiting severity is graded by the patient. More recent measures of vomiting have assessed the frequency and duration of vomiting episodes, instead of the severity of vomiting. Future studies should take these aspects into consideration in assessing vomiting severity. We did not find a relationship of worsening glucose control in diabetic patients with different severities of nausea or with vomiting. Autonomic dysfunction sometimes present in diabetic patients may be related to the presence of vomiting. Vagal and non-vagal pathways and several brainstem nuclei participate in vomiting in response to different emetic stimuli. ${ }^{22}$ Physiologic differences between idiopathic and DG may relate to worse vagal impairments in diabetics. ${ }^{23,24}$

Each of the symptoms of the nausea/vomiting subscore (nausea, retching, vomiting) tracked with each other. In addition, increasing nausea and increasing vomiting were related to increasing satiety/fullness subscore and upper abdominal pain; this is not surprising as these are the symptoms of gastroparesis. Satiety severity associated with nausea severity suggests a vagal neuropathy as a possible cause. We also found that as nausea and vomiting increased, there was an increased use of antiemetic agents as expected but also the use of narcotic analgesics. Narcotics can delay gastric emptying and cause nausea and vomiting as a side effect. Our study demonstrates a relationship of narcotics with symptoms but not with delayed gastric emptying. The relation of narcotic analgesics with nausea might be related to the central effects of opiates rather than their peripheral effects in slowing gastric emptying.

The results of the study show that nausea and vomiting severity varies by gastric emptying but are not linearly related. Our study showed increased severity of nausea among severely delayed gastric emptying. However, the statistical trend test for both nausea and vomiting showed no significant systematic relationship with gastric emptying ( $p=0.09$ for both). In a previous study from our GpCRC, we did not show a significant relationship between nausea severity and delay of gastric emptying. ${ }^{25}$ This study included assessment of gastric emptying using liquids as well; however, we found that the liquid results were consistent with the solid results - we did not find a relationship between retention of liquids and symptom severity of nausea or vomiting. The symptom assessment at enrollment was not on the same day as the gastric emptying test (median separation of 13 days, IQR 0-95 days); however, even when symptoms at the time of gastric emptying were assessed, only stomach fullness, but not nausea or vomiting, appeared to be associated with increasing delay in gastric emptying.

In conclusion, this study demonstrates that nausea and vomiting are important symptoms of gastroparesis. The severity of nausea is related to the decrease in quality of life that is present in patients with gastroparesis. Characteristics of nausea appeared similar between diabetic and IG. Vomiting, however, was more prevalent and severe in DG than in IG, occurred more often in the morning in DG, during the night and when not eating. Thus, although characteristics of nausea appear to be similar between diabetic and IG, the characteristics of vomiting differ in idiopathic vs DG. Symptoms of nausea and vomiting are important symptoms that need to be specifically addressed, perhaps individually, in treating patients with gastroparesis.

\section{FUNDING}

The Gastroparesis Clinical Research Consortium (GpCRC) is supported by the National Institute of Diabetes and Digestive and Kidney Diseases (NIDDK) (grants U01DK073983, U01DK073975, U01DK073985, U01DK074007, U01DK073974, U01DK074008). 


\section{CONFLICTS OF INTEREST}

No conflicts of interest exist.

\section{AUTHOR CONTRIBUTION}

HPP performed study conceptualization, patient recruitment, data interpretation, and writing the manuscript; EH was responsible for statistical analysis, data interpretation, and writing the manuscript; WLH, KLK, WJS, TLA, IS, LN, and PJP contributed to study conceptualization, patient recruitment, and revising the manuscript; GF, JC, LM, LL, and FH were involved in study conceptualization and revising the manuscript; RWM and JC carried out patient recruitment, and revising the manuscript; JT was responsible for study conceptualization, statistical analysis, data interpretation, and revising the manuscript.

\section{REFERENCES}

1 Parkman HP, Hasler WL, Fisher RS. American Gastroenterological Association technical review on the diagnosis and treatment of gastroparesis. Gastroenterology 2004; 127: 1592-622.

2 Soykan I, Sivri B, Sarosiek I, Kiernan B, McCallum RW. Demography, clinical characteristics, psychological and abuse profiles, treatment, and long-term follow-up of patients with gastroparesis. Dig Dis Sci 1998; 43: 2398-404.

3 Jaffe JK, Paladugu S, Gaughan JP, Parkman HP. Characteristics of nausea and its effects on quality of life in diabetic and idiopathic gastroparesis. $I$ Clin Gastroenterol 2011; 45: 317-21.

4 Cherian D, Parkman HP. Nausea and vomiting in diabetic and idiopathic gastroparesis. Neurogastroenterol Motil 2012; 24: 217-22.

5 Parkman HP, Yates K, Hasler WL, Nguyen L, Pasricha PI, Snape WJ, Farrugia G, Koch KL et al. Clinical features of idiopathic gastroparesis vary with sex, body mass, symptom onset, delay in gastric emptying, and gastroparesis severity. Gastroenterology 2011; 140: 101-15.

6 Hasler WL, Parkman HP, Wilson LA, Pasricha PJ, Koch KL, Abell TL, Snape WJ, Farrugia G et al. Psychological dysfunction is associated with symptom severity but not disease etiology or degree of gastric retention in patients with gastroparesis. Am I Gastroenterol 2010; 105: 2357-67.

7 Abell TL, Bernstein RK, Cutts T, Farrugia G, Forster I, Hasler WL, McCallum RW, Olden KW et al. Treatment of gastroparesis: a multidisciplinary clinical review. Neurogastroenterol Motil 2006; 18: 263-83.

8 Rentz AM, Kahrilas P, Stanghellini V, Tack J, Talley NJ, de la Loge C, Trudeau E, Dubois D et al. Development and psychometric evaluation of the patient assessment of upper gas- trointestinal symptom severity index (PAGI-SYM) in patients with upper gastrointestinal disorders. Qual Life Res 2004; 13: 1737-49.

9 Revicki DA, Rentz AM, Dubois D, Kahrilas P, Stanghellini V, Talley NJ Tack J. Development and validation of a patient-assessed gastroparesis symptom severity measure: the Gastroparesis Cardinal Symptom Index. Aliment Pharmacol Ther 2003; 18: 141-50.

10 De la Loge C, Trudeau E, Marquis P, Kahrilas P, Stanghellini V, Talley NJ, Tack J, Revicki DA et al. Crosscultural development and validation of a patient self-administered questionnaire to assess quality of life in upper gastrointestinal disorders: the PAGI-QOL. Qual Life Res 2004; 13: 1751-62.

11 Ware JE, Kosinski M, Dewey JE. How to Score Version 2 of the SF-36 ${ }^{\circledR}$ Health Survey. Lincoln, RI: QualityMetric Incorporated, 2000.

12 Muth ER, Stern RM, Thayer JF, Koch KL. Assessment of the multiple dimensions of nausea: the Nausea Profile (NP). I Psychosom Res 1996; 40: 511-20.

13 Tougas G, Eaker EY, Abell TL, Abrahamsson $H$, Boivin $M$, Chen J, Hocking MP, Quigley EM et al. Assessment of gastric emptying using a low fat meal: establishment of international control values. Am I Gastroenterol 2000; 95: 1456-62.

14 Abell TL, Camilleri M, Donohoe K, Hasler WL, Lin HC, Maurer AH, McCallum RW, Nowak T et al. Consensus recommendations for gastric emptying scintigraphy. Am I Gastroenterol 2008; 103: 753-63.

15 Sachdeva P, Malhotra N, Pathikonda M, Khayyam U, Fisher RS, Maurer AH, Parkman HP. Gastric emptying of solids and liquids for evaluation for gastroparesis. Dig Dis Sci 2011; 56: 1138-46.
16 Camilleri M. Diabetic gastroparesis. N Engl J Med 2007; 356: 820-9.

17 Agresti A. Categorical Data Analysis. New York: John Wiley \& Sons, Inc., 1990.

18 Akaike H. A new look at the statistical model identification. IEEE Trans Autom Control 1974; 19: 716-23.

19 Hosmer D, Lemeshow S. Applied Logistic Regression, 2nd edn. New York: John Wiley \& Sons, Inc., 2000.

20 SAS Institute, Inc. SAS software, version 9.3 of the SAS system for Windows. Cary, NC, 2002-2010. Stata statistical software: release 12 . College Station, TX: StataCorp LP, 2011.

21 Parkman HP, Yates K, Hasler WL, Nguyen L, Pasricha PJ, Snape WI, Farrugia G, Koch KL et al. Similarities and differences between diabetic and idiopathic gastroparesis. Gastroenterology 2011; 9: 1056-64.

22 Hasler WL. Pathology of emesis: its autonomic basis. Handb Clin Neurol 2013; 117: 337-52.

23 Simonian HP, Kresge KM, Boden GH, Parkman HP. Differential effects of sham feeding and meal ingestion on ghrelin and pancreatic polypeptide levels: evidence for vagal efferent stimulation mediating ghrelin release. Neurogastroenterol Motil 2005; 17: 348-54.

24 Hasler WL, Coleski R, Chey WD, Koch KL, McCallum RW, Wo JM, Kuo B, Sitrin MD, Katz LA et al. Differences in intragastric $\mathrm{pH}$ in diabetic versus idiopathic gastroparesis: relation to degree of gastric retention. Am I Physiol 2008; 294: G1384-91.

25 Pasricha PJ, Colvin R, Yates K, Hasler WL, Abell TL, Ünalp-Arida A, Nguyen L, Farrugia G et al. Characteristics of patients with chronic unexplained nausea and vomiting and normal gastric emptying. Clin Gastroenterol Hepatol 2011; 9: 567-76.e1-4. 


\section{SUPPORTING INFORMATION}

Additional Supporting Information may be found online in the supporting information tab for this article:

Table S1 Baseline characteristics of patients with idiopathic or diabetic gastroparesis by $4 \mathrm{~h}$ gastric retention.

Table S2 Nausea and vomiting profile of patients with idiopathic or diabetic gastroparesis. 